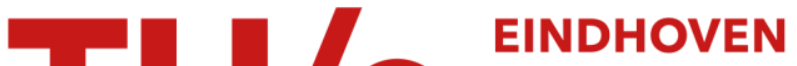 UNIVERSITY OF TECHNOLOGY
}

\section{Efficient Brownian dynamics simulation of particles near walls. II. Sticky walls}

\section{Citation for published version (APA):}

Peters, E. A. J. F., \& Barenbrug, T. M. A. O. M. (2002). Efficient Brownian dynamics simulation of particles near walls. II. Sticky walls. Physical Review E - Statistical, Nonlinear, and Soft Matter Physics, 66(5), 056702-1/11.

[056702]. https://doi.org/10.1103/PhysRevE.66.056702

DOI:

10.1103/PhysRevE.66.056702

Document status and date:

Published: 01/01/2002

\section{Document Version:}

Publisher's PDF, also known as Version of Record (includes final page, issue and volume numbers)

\section{Please check the document version of this publication:}

- A submitted manuscript is the version of the article upon submission and before peer-review. There can be important differences between the submitted version and the official published version of record. People interested in the research are advised to contact the author for the final version of the publication, or visit the $\mathrm{DOI}$ to the publisher's website.

- The final author version and the galley proof are versions of the publication after peer review.

- The final published version features the final layout of the paper including the volume, issue and page numbers.

Link to publication

\section{General rights}

Copyright and moral rights for the publications made accessible in the public portal are retained by the authors and/or other copyright owners and it is a condition of accessing publications that users recognise and abide by the legal requirements associated with these rights.

- Users may download and print one copy of any publication from the public portal for the purpose of private study or research.

- You may not further distribute the material or use it for any profit-making activity or commercial gain

- You may freely distribute the URL identifying the publication in the public portal.

If the publication is distributed under the terms of Article 25fa of the Dutch Copyright Act, indicated by the "Taverne" license above, please follow below link for the End User Agreement:

www.tue.nl/taverne

Take down policy

If you believe that this document breaches copyright please contact us at:

openaccess@tue.nl

providing details and we will investigate your claim. 


\title{
Efficient Brownian dynamics simulation of particles near walls. II. Sticky walls
}

\author{
E. A. J. F. Peters* \\ Department of Chemical Engineering, Universiteit van Amsterdam, Nieuwe Achtergracht 166, 1018WV Amsterdam, The Netherlands
}

Th. M. A. O. M. Barenbrug

Department of Chemical Engineering, Università degli Studi di Napoli Frederico II, Piazzale V. Tecchio 80, 80125 Napoli, Italy

(Received 5 June 2002; published 6 November 2002)

\begin{abstract}
In this paper we treat a boundary condition, the "sticky boundary," which appears to be quite useful in mesoscopic models. The sticky boundary is modeled as an infinitely deep, infinitely narrow, potential well adjacent to a reflecting boundary. The free energy corresponding to this boundary is finite. The boundary condition, which can be viewed as an intermediate between the absorbing and reflecting boundary condition, may have many applications, e.g., for the simulation of the partial adsorption of polymer molecules to walls and for the modeling of solvent quality. We will derive an efficient Brownian dynamics algorithm, capable of handling interactions of a diffusing particle with a sticky wall. Our approach avoids the large discretization errors that occur in the simulation of boundary interactions within the "standard" Brownian dynamics approach. The essence of our method was presented before [E. Peters and T. Barenbrug, Phys. Rev. E (to be published)]. The treatment of the wall as proposed here is quite general, and therefore not limited to the use within Brownian dynamics. In other simulation techniques which aim at treating the dynamics of mesoscopic particles near walls, we expect it to be of use as well.
\end{abstract}

DOI: 10.1103/PhysRevE.66.056702

PACS number(s): 02.70.Ns, 05.40.-a, 02.50.Ey, 68.43.-h

\section{INTRODUCTION}

In this paper we discuss the "sticky" boundary condition. We assume that the "stickiness" of the wall can be described by a narrow but deep potential well, adjacent to a reflecting wall. If a particle resides in this well, we say it "sticks to the wall." Otherwise the particle is called "free." The wall itself obeys reflecting boundary conditions. The width of the well is assumed to be much less than the typical displacement of the Brownian particle during the chosen time step. However, the width of the well must be sufficiently large and spatial variations in the potential must be slow enough, compared to the particle's free path, so that the Brownian description is valid everywhere. Furthermore, the potential is assumed to have no long-range contributions.

For any potential field $U(x)$, the equilibrium probability density distribution of a particle in this field is given by the well-known Boltzmann distribution

$$
p^{\mathrm{EQ}}(x)=\frac{1}{Z} \exp \left[-\frac{U(x)}{k T}\right]
$$

where $Z$, the total sum of states, is a normalization factor. Since we assume that the potential well is narrow compared to the length scales resolved by the description, this full probability distribution is not a very useful quantity. Much more interesting is the total probability that a particle sticks to the wall, i.e., resides somewhere in the potential well. This probability can be found by integrating over the width of the well, denoted by $b$,

\footnotetext{
*Electronic address: fpeters@science.uva.nl
}

$$
P_{\text {wall }}^{\mathrm{EQ}}=\frac{1}{Z} \int_{0}^{b} \exp \left[-\frac{U(x)}{k T}\right] d x .
$$

Note that throughout this paper we denote a probability by " $P$," and a probability distribution by " $p$." If we define the "wall free energy" by

$$
F_{\text {wall }}=-k T \ln \left\{\int_{0}^{b} \exp \left[-\frac{U(x)}{k T}\right] d x\right\},
$$

the above probability can be expressed as

$$
P_{\text {wall }}^{\mathrm{EQ}}=\frac{1}{Z} \exp \left[-\frac{F_{\text {wall }}}{k T}\right]=p_{\text {free }}^{\mathrm{EQ}} \exp \left[-\frac{F_{\text {wall }}}{k T}\right],
$$

where the last equality is a simple result of Eq. (1) with $U$ $=0$. We will discuss some implications of Eq. (3) later.

We are interested in phenomena on length scales much larger than $b$. We postulate that in this regime the wall free energy $F_{\text {wall }}$ is sufficient to describe the wall interaction. For equilibrium properties this is obviously true, whereas for the dynamical ones it is less obvious. On the one hand, one could argue that by changing both $E$ and $b$, while leaving $F_{\text {wall }}$ unchanged, quantities such as escape rate might change. On the other hand, it is unlikely that such a change of the internal variables of the well would influence the entrapment rate, under the assumption that the Brownian moves of the particle are much larger than $b$. If only the escape rate is influenced while the entrapment rate remains the same, the equilibrium statistics would change by this operation. Since the equilibrium state does not change when $F_{\text {wall }}$ is kept constant, the dynamics of the system cannot change either. We therefore draw the conclusion that in the regime of relatively large (time) steps the dynamics of the 
system are governed by $F_{\text {wall }}$ only. Note that this argument is a purely qualitative one. In fact, in the pure diffusion regime considered here one cannot even unambiguously define escape and entrapment rates. We will return to this point further on.

\section{WALL INTERACTION DYNAMICS}

Building on the above arguments we postulate that only $F_{\text {wall }}$ is important for the description of the dynamics on length scales much larger than the width, $b$, of the potential well. Therefore one has some freedom in choosing the function $U(x)$. We make the convenient choice of a square well potential,

$$
U(x)= \begin{cases}-E & \text { for } x<b \\ 0 & \text { for } x>b\end{cases}
$$

In the preceding section we already stressed the fact that the wall potential should be sufficiently smooth for the Brownian description to be valid everywhere. Therefore, this potential should be understood as a mathematically convenient approximation of such a smooth potential. Then the wall free energy is given [see Eq. (3)] by

$$
F_{\text {wall }}=-E-k T \ln b .
$$

In our previous paper [1], we pointed out the reason for the large discretization error of naive algorithms used for the simulation of Brownian particles in the vicinity of walls: the systematic and substantial underestimation of diffusive interactions with the wall within every time step of the Brownian dynamics algorithm. The solution of this problem is straightforward. A correct discretization of the stochastic differential equation (SDE) consists of the generation of displacements according to a conditional probability distribution that approximates the true distribution up to sufficient order in the time step. For the particle near a flat absorbing or reflecting wall, it was shown that any distribution with the correct zeroth, first, and second moments (which does not cross the boundary) gives a correct discretization with a global accuracy of $O(\Delta t)$.

In this paper essentially the same is done, although the situation has become more complicated. Again the numerical approximation to the SDE for the particle must quantitatively contain all relevant aspects of its motion, but now with respect to the sticky wall. Due to the presence of the potential well, these aspects now must include not only displacements, but also the possible transitions, depending on its starting position, e.g., remaining stuck to the wall, being attached during the time step, being detached, and remaining free.

The central quantity needed for the calculation of these probabilities and probability densities for the motion and transitions of the Brownian particle with respect to the sticky wall, is the Green function, $G\left(\Delta t, x, x_{0}\right)$, associated with the chosen system. In Appendix A an expression for this Green function is derived. By means of this expression, the different transition probabilities and probability densities are derived in the following two sections. As the last step in these derivations, we take the limits $b \rightarrow 0$ and $E \rightarrow \infty$, while keeping $F_{\text {wall }}$ constant, to arrive at expressions that are appropriate for our model of a "sticky wall." For all computations we use $k T=1$ and $D=1$. This does not make the equations fully dimensionless. Later we will also introduce a characteristic time $t_{\text {char }}$, which will be related to a characteristic length scale $x_{\text {char }}$ by means of the diffusion constant.

Additionally, in Appendix B we have considered this same limiting case, to construct the appropriate sticky boundary condition for the diffusion equation. The solution of the Fokker-Planck equation with this boundary condition could be used for an alternative approach to the Brownian dynamics algorithm as presented here.

\section{A. Sticking probabilities}

First we consider the probability that a particle that sticked to the wall at time $t=0$ also sticks to the wall at time $t=\Delta t$. The resulting probability includes possible intermediate states in which the diffusing particle is free and/or sticking within the chosen time step. The calculated probabilities solely refer to the situations at the start and at the end of the time step.

Before the limit $b \rightarrow 0$ is taken, a sticking particle is characterized by the fact that it resides in the well: $x_{0}<b$. The chance that a particle also sticks at time $t=\Delta t$ is equivalent to the probability that it resides at any position $x<b$ at that time. Using the Green function, Eq. (A25), we therefore find

$$
\begin{aligned}
P_{\text {stick } \rightarrow \text { stick }}(\Delta t)= & \lim _{b \rightarrow 0} \int_{0}^{b} G\left(\Delta t, x, x_{0}\right) d x \text { with } x_{0}<b \\
= & \frac{2}{\pi_{b \rightarrow 0}} \lim _{0}^{\infty} \frac{\exp [-E] \sin \lambda b \cos \lambda x_{0}}{\lambda\left(\sin ^{2} \lambda b+\exp [-2 E] \cos ^{2} \lambda b\right)} \\
& \times \exp \left[-\lambda^{2} \Delta t\right] d \lambda \\
= & \frac{2}{\pi} \int_{0}^{\infty} \frac{\exp \left[F_{\text {wall }}\right]}{\lambda^{2}+\exp \left[2 F_{\text {wall }}\right]} \exp \left[-\lambda^{2} \Delta t\right] d \lambda \\
= & \frac{2}{\pi} \int_{0}^{\infty} \frac{1}{\tilde{\lambda^{2}+1} \exp \left[-\tilde{\lambda}{ }^{2} \Delta t \exp \left(2 F_{\text {wall }}\right)\right] d \tilde{\lambda}} \\
= & \exp \left[\Delta t \exp \left(2 F_{\text {wall }}\right)\right] \operatorname{erfc}\left[\sqrt{\Delta t \exp \left(2 F_{\text {wall }}\right)}\right] \\
= & \exp [\Delta \tilde{t}] \operatorname{erfc}[\sqrt{\Delta \tilde{t}]},
\end{aligned}
$$

where we first performed the integral over $x$, then substituted Eq. (3) for the wall free energy and then took the limit $b$ $\rightarrow 0$. Note that the $x_{0}$ dependence drops out when taking the limit $b \rightarrow 0$, which means that the statement: "at the wall" is unambiguous in this limit. The function erfc[] is the complementary error function, i.e., $\operatorname{erfc}[]=1-\operatorname{erf}[]$.

The symbols indicated by the tilde are made dimensionless by means of the characteristic time and the characteristic length scale, by means of 


$$
\tilde{\lambda}=\lambda x_{\text {char }}, \quad \tilde{t}=t / t_{\text {char }}, \quad \tilde{x}=x / x_{\text {char }} .
$$

The characteristic length scale is given by

$$
x_{\text {char }}=\exp \left[-F_{\text {wall }} / k T\right] .
$$

At first sight one might think there is something wrong concerning the dimensions in this equation. This is not the case. A careful look at $\exp \left[-F_{\text {wall }} / k T\right]$, using Eq. (6), reveals that this quantity has the dimension of length. The cause for this is the definition of $F_{\text {wall }}$ in Eq. (3). Here we take a logarithm of a dimensional quantity (with dimension length). This could have been prevented by computing a full partition function of the wall region and not only the configurational part. In this way one should integrate the full Boltzmann factor also over momentum space and divide by the elementary volume of phase space, which is given by quantum mechanics to be the Planck constant $h$. This procedure would give rise to the normalization of the integral within the logarithm by a thermal de Broglie wavelength $\lambda$. This wavelength has no physical significance in our problem. The full free energy is only changed by a constant offset compared to the expression we use here. Using the full expression of the free energy and $\lambda$ one can construct a characteristic length scale for the problem (which is of course independent of $\lambda$ ). This length scale is exactly the same characteristic length scale as the one given by Eq. (9). Note that the free energy defined by Eq. 3 is not ill defined in a mathematical sense. The reason is that the multiplication of units and numbers (or other units) is communicative. Therefore, the ordinary rules for mathematical functions such as the logarithm are obeyed for these quantities.

The characteristic time $t_{\text {char }}$ is defined as the mean time needed to diffuse a distance $x_{\text {char }}$, i.e.,

$$
t_{\text {char }}=\frac{x_{\text {char }}^{2}}{D}=\frac{\exp \left[-2 F_{\text {wall }} / k T\right]}{D} .
$$

Note that $t_{\text {char }}$ does not have the well-known Arrhenius shape. In the case of the Arrhenius scaling the time scale is the Boltzmann factor divided by a collision frequency. This scaling is only valid if the width of the potential barrier is smaller than the mean free path. If a particle approaches the barrier and has a sufficiently large kinetic energy it will escape. In our case, however, we are in the diffusion regime. The width of the real physical barrier (i.e., as opposed to our square potential approximation) is much wider than the particle's free path. Therefore the momentum of the particle reequilibrates before the barrier is fully crossed. It will not be crossed in a ballistic manner. This kind of wall interaction is probably a good approximation for many surface phenomena in liquid or soft-condensed systems.

In the Brownian regime the particle trajectory remains a fractal object. Looking at the sticky wall, the smaller the time step one chooses, the more escapes and entrapments one will find in a certain fixed period of time. However, in most cases the escaped particle will stick to the wall again after the next time step. Therefore, these escapes remain unnoticed if one uses a larger time step. Counting escapes and entrapments as such is therefore not very useful.

For the limiting cases of very small and very large time steps, one can find simple approximations,

$$
P_{\text {stick } \rightarrow \operatorname{sick}}(\Delta t) \approx \begin{cases}1-2 \sqrt{\frac{\Delta \tilde{t}}{\pi}} & \text { for } \Delta t \ll t_{\text {char }}, \\ \sqrt{\frac{t_{\text {char }}}{\pi \Delta \tilde{t}}} & \text { for } \Delta t \gg t_{\text {char }} .\end{cases}
$$

For $\Delta t=0$ the time derivative does not exist, which illustrates that no (initial) escape rate can be defined.

The second case we consider is the probability that a particle in free space at time 0 will be entrapped by the wall at time $\Delta t$. This probability is given by

$$
\begin{aligned}
P_{\text {free } \rightarrow \text { stick }}\left(\Delta t, x_{0}\right)= & \lim _{b \rightarrow 0} \int_{0}^{b} G\left(\Delta t, x, x_{0}\right) d x, \text { with } x<b \\
= & \frac{2}{\pi_{b \rightarrow 0}} \int_{0}^{\infty} \frac{\sin \lambda b}{\lambda} \frac{\left(\sin ^{2} \lambda b+\exp [-2 E] \cos ^{2} \lambda b\right) \cos \lambda x_{0}-\{\sin \lambda b \cos \lambda b(1-\exp [-E])\} \lambda \sin \lambda x_{0}}{\sin ^{2} \lambda b+\exp [-2 E] \cos ^{2} \lambda b} \\
& \times \exp \left(-\lambda^{2} \Delta t\right) d \lambda \\
= & \frac{2}{\pi} \int_{0}^{\infty} \frac{\cos \left(\tilde{\lambda} \exp \left[F_{\text {wall }}\right] x_{0}\right)-\tilde{\lambda} \sin \left(\tilde{\lambda} \exp \left[F_{\text {wall }}\right] x_{0}\right)}{\tilde{\lambda^{2}}+1} \exp \left[-\tilde{\lambda}^{2} \exp \left(2 F_{\text {wall }}\right) \Delta t\right] d \tilde{\lambda} \\
= & \exp \left[\tilde{x}_{0}+\Delta \tilde{t}\right] \operatorname{erfc}\left[\frac{\tilde{x}_{0}+2 \Delta \tilde{t}}{2 \sqrt{\Delta \tilde{t}}]}\right.
\end{aligned}
$$


where we first performed the integral over $x$, then substituted Eq. (3) for the wall free energy and then took the limit $b$ $\rightarrow 0$. In Fig. 1, $P_{\text {free } \rightarrow \text { stick }}\left(\Delta t, x_{0}\right)$ is plotted as a function of dimensionless time $\Delta t / t_{\text {char }}$, for several values of $x_{0}$.

In the limit $x_{0} \rightarrow 0$ one finds that $P_{\text {free } \rightarrow \text { stick }}\left(\Delta t, x_{0}\right)$ approaches $P_{\text {stick } \rightarrow \text { stick }}(\Delta t)$. This should be the case, since very close to the wall the probability of entrapment approaches 1 . Once entrapped, a particle behaves like a sticking particle, so that the limit $x_{0} \rightarrow 0$ behaves in a continuous way.

In Fig. 2 the spatial dependence of $P_{\text {free } \rightarrow \text { stick }}\left(\Delta t, x_{0}\right)$ is plotted, where the position is made dimensionless by means of division by $\sqrt{D \Delta t}$, which is a measure for average displacement due to diffusive motion in a time $\Delta t$. From this graph it becomes clear that, in order to describe the wall interaction in a time step correctly, one only needs to consider the region within a few distances $\sqrt{D \Delta t}$ from the wall. In the left graph $P_{\text {free } \rightarrow \text { stick }}\left(\Delta t, x_{0}\right)$ is shown. In the right graph a multiplication factor of $\sqrt{\pi \Delta t / t_{\text {char }}}$ is used, corresponding to the limiting short and long time behavior. The short time behavior corresponds to the situation of a fully absorbing wall $P_{\text {free } \rightarrow \text { absorb }}\left(\Delta t, x_{0}\right)=\operatorname{erfc}[x /(2 \sqrt{D \Delta t})]$. For long times the fully reflecting case is approached. In the perfectly reflecting case, of course, no particle will stick to the wall. Below we discuss the close relationship that exists between the results for a sticky and a fully reflecting wall.

\section{B. Probability distributions in free space}

In this section we study the cases opposite to those discussed in the preceding section: particles of which the final position is not at the wall, but in free space. The state at the wall is macroscopically occupied and can therefore be described in terms of probabilities. The state in free space must be described in terms of probability densities. Again there are two cases to be considered. The first is the situation where the particle starts at the wall and ends up in free space.

For the particle that starts at the wall, the initial conditions are $x>b$ and $x_{0}<b$. In this situation the Green's function, Eq. (A25), in the limit of $b \rightarrow 0$ and a fixed value of $F_{\text {wall }}$ reduces to

$$
\begin{aligned}
p_{\text {stick } \rightarrow \text { free }}(\Delta t, x) & =\lim _{b \rightarrow 0} G\left(\Delta t, x, x_{0}\right) \\
& =\frac{2}{\pi} \exp \left[F_{\text {wall }}\right] \int_{0}^{\infty} \frac{\exp \left[F_{\text {wall }}\right] \cos \lambda x-\lambda \sin \lambda x}{\lambda^{2}+\exp \left[2 F_{\text {wall }}\right]} \exp \left[-\lambda^{2} \Delta t\right] d \lambda \\
& =\frac{2}{\pi} \exp \left[F_{\text {wall }}\right] \int_{0}^{\infty} \frac{\cos \left(\tilde{\lambda} \exp \left[F_{\text {wall }}\right] x\right)-\tilde{\lambda} \sin \left(\tilde{\lambda} \exp \left[F_{\text {wall }}\right] x\right)}{\tilde{\lambda}^{2}+1} \exp \left[-\tilde{\lambda}^{2} \exp \left(2 F_{\text {wall }}\right) \Delta t\right] d \tilde{\lambda} \\
& =\frac{1}{x_{\text {char }}} \exp [\tilde{x}+\Delta \tilde{t}] \operatorname{erfc}\left[\frac{\tilde{x}+2 \Delta \tilde{t}}{2 \sqrt{\Delta \tilde{t}}}\right] .
\end{aligned}
$$

One can check (by partial integration) that the total probability is correctly normalized, e.g.,

$$
\int_{0}^{\infty} p_{\text {stick } \rightarrow \text { free }}(\Delta t, x) d x=1-P_{\text {stick } \rightarrow \text { stick }}(\Delta t) .
$$

When inspecting Eq. (6) once more, one can conclude that a sticky wall with characteristic length $x_{\text {char }}$ is equivalent (in its long time behavior) to a "degenerate" potential well of width $x_{\text {char }}$ and no energy difference, i.e., $E=0$ and $b$ $=x_{\text {char }}$ in Eq. (6). The latter system can be understood as if behind the original well, at a distance $x_{\text {char }}$, a virtual wall is placed, with fully reflecting properties. This wall, together with this extra distance, appears to (almost) replace the effect of the potential well, at least for larger diffusion times. The probability distribution for a particle released near the virtual reflecting wall, would be

$$
p_{\text {approx }}(\Delta t, x)=\frac{1}{\sqrt{\pi D \Delta t}} \exp \left[\frac{x+x_{\text {char }}}{4 D \Delta t}\right] .
$$

In Fig. 3 we compare this approximation with the analytic solution Eq. (13) and see that the comparison is very good for longer times. This shows that $t_{\text {char }}$, i.e., the time the particle needs to travel the virtual distance behind the wall, can be considered a kind of retardation time, i.e., the average time the sticky wall succeeds in capturing a particle, after which it is able to escape and continue its journey as a free particle. For really long times, when $x_{\text {char }} \ll \sqrt{D \Delta t}$, the limiting curve is also approximated well by a Gaussian curve with the center at $x=0$ (instead of $x=x_{\text {char }}$ ).

Expression Eq. (13) is almost equal to that of $P_{\text {free } \rightarrow \text { stick }}$, Eq. (12). The only difference is the occurrence of the term $\exp \left[F_{\text {wall }}\right]$. This observation explains why the long time limiting behavior in the right graph of Fig. 2 is that of a Gaussian curve. The relation can be understood by looking at the 
equilibrium situation. In case of equilibrium, detailed balance is obeyed between the position at the wall and any point in space. This means that the probability density for a particle to be at the wall and to end up at a position $x$ after one time step equals the probability for the opposite situation,

$P_{\text {wall }}^{\mathrm{EQ}} p_{\text {stick } \rightarrow \text { free }}(\Delta t, x)=p_{\text {free }}^{\mathrm{EQ}} P_{\text {free } \rightarrow \text { stick }}(\Delta t, x)$, substituting Eq. (4) gives the relationship found.

The last probability distribution needed is that of a free particle that, after a time step, is also free $\left(x_{0}>b\right.$ and $x$ $>b$ ). Again, one should note that this does not exclude the possibility that at a certain intermediate moment in time the particle has been sticking to the wall. This probability density is given by

$$
\begin{aligned}
& p_{\text {free } \rightarrow \text { free }}\left(\Delta t, x, x_{0}\right)=\lim _{b \rightarrow 0} G\left(\Delta t, x, x_{0}\right) \\
& =\frac{2}{\pi} \exp \left[F_{\text {wall }}\right] \\
& \times \int_{0}^{\infty} \frac{\left\{\cos \left(\tilde{\lambda} \exp \left[F_{\text {wall }}\right] x\right)-\tilde{\lambda} \sin \left(\tilde{\lambda} \exp \left[F_{\text {wall }}\right] x\right)\right\} \times\left\{\cos \left(\tilde{\lambda} \exp \left[F_{\text {wall }}\right] x_{0}\right)-\tilde{\lambda} \sin \left(\tilde{\lambda} \exp \left[F_{\text {wall }}\right] x_{0}\right)\right\}}{\tilde{\lambda}^{2}+1} \\
& \times \exp \left[-\widetilde{\lambda}^{2} \exp \left(2 F_{\text {wall }}\right) \Delta t\right] d \tilde{\lambda} \\
& =\frac{1}{x_{\text {char }}} \exp \left[\tilde{x}+\tilde{x}_{0}+\Delta \tilde{t}\right] \operatorname{erfc}\left[\frac{\tilde{x}+\tilde{x}_{0}+2 \Delta \tilde{t}}{2 \sqrt{\Delta \tilde{t}}}\right]+\frac{1}{x_{\text {char }}} \frac{1}{2 \sqrt{\pi \Delta \tilde{t}}}\left(\exp \left[-\frac{\left(\tilde{x}-\tilde{x}_{0}\right)^{2}}{4 \Delta \tilde{t}}\right]-\exp \left[-\frac{\left(\tilde{x}+\tilde{x}_{0}\right)^{2}}{4 \Delta \tilde{t}}\right]\right) \text {. }
\end{aligned}
$$

The second contribution in the last expression, i.e., the antisymmetrized Gaussian, is the "survival" probability density one would obtain in the case of an absorbing wall. It, therefore, denotes the probability density for the case that there is no interaction with the wall within a time step $\Delta t$. The first contribution is thus the probability distribution for the case that there is at least one interaction with the wall. This contribution is equal to that of the particle starting at the wall, Eq. (13), with $\tilde{x}_{0}+\tilde{x}$ substituted for $\tilde{x}$. This can be interpreted as follows. If one knows there is at least one interaction with the wall, the particle has to diffuse over a distance $x_{0}$ to reach the wall, and subsequently a distance $x$ from the wall to the final position, thus a total distance $x+x_{0}$. For the probability density it does not matter where in the interval of length $x+x_{0}$ the interaction with the wall takes place. Therefore, the probability distribution for the wall interaction at the initial position equals that of interaction somewhere within this interval.

\section{THE SIMULATION ALGORITHM}

In the system of Brownian particles in the neighborhood of a sticky wall, one has to account for two kinds of particles, namely, the particles that are sticking to the wall and the free particles. Due to the substantial stickiness of the wall, it will be macroscopically occupied by sticking particles. In a Brownian dynamics simulation of particles near (or on) a sticky wall, at every time step two operations have to be performed. For each of the particles one has to check whether it will stick at the wall after the time step. If this is not the case, the new position of the particle in (free) space has to be determined.

The probability that a particle, initially located at $x_{0}$, will end up at the wall after a time $\Delta t$ is $P_{\text {free } \rightarrow \text { stick }}\left(\Delta t, x_{0}\right.$ ) (where $x_{0}=0$ corresponds to a particle initially on the wall). To determine whether this particle will stick at the wall after the time step, we draw a random number between 0 and 1 . If this number is less than $P_{\text {free } \rightarrow \text { stick }}\left(\Delta t, x_{0}\right)$ it will be located at the wall after the time step. Otherwise it is considered free after the time step.

If the particle turns out to be free, we have to perform a random move. As explained in Ref. [1], for a valid $[O(\Delta t)]$ Brownian dynamics algorithm only the zeroth, first, and second moments of the probability distribution of the particle's displacement are needed, i.e.,

$$
\left\langle\tilde{x}^{0}\right\rangle=\int_{0}^{\infty} p_{\text {free } \rightarrow \text { free }}\left(\Delta \tilde{t}, \tilde{x}, \tilde{x}_{0}\right) d \tilde{x}=1-P_{\text {free } \rightarrow \text { stick }}\left(\Delta \tilde{t}, \tilde{x}_{0}\right),
$$

$$
\begin{aligned}
& \left\langle\tilde{x}^{1}\right\rangle=\int_{0}^{\infty} \tilde{x} p_{\text {free } \rightarrow \text { free }}\left(\Delta \tilde{t}, \tilde{x}, \tilde{x}_{0}\right) d \tilde{x} \\
& =-1+2 \sqrt{\frac{t}{\pi} \exp }\left[-\frac{\tilde{x}_{0}^{2}}{4 \Delta \tilde{t}}\right]+\left(1+\tilde{x}_{0}\right) \operatorname{erf}\left[\frac{\tilde{x}_{0}}{2 \sqrt{\Delta \tilde{t}}}\right] \\
& +\exp \left[\Delta \tilde{t}+\tilde{x}_{0}\right] \operatorname{erfc}\left[\frac{2 t+\tilde{x}_{0}}{2 \sqrt{\Delta \tilde{t}}}\right]
\end{aligned}
$$




$$
\begin{aligned}
\left\langle\tilde{x}^{2}\right\rangle= & \int_{0}^{\infty} \tilde{x}^{2} p_{\text {free } \rightarrow \text { free }}\left(\Delta \tilde{t}, \tilde{x}, \tilde{x}_{0}\right) d \tilde{x} \\
= & 2-4 \sqrt{\frac{\Delta \tilde{t}}{\pi} \exp \left[-\frac{\tilde{x}_{0}^{2}}{4 \Delta \tilde{t}}\right]+2 \Delta \tilde{t}+\tilde{x}_{0}\left(2+\tilde{x}_{0}\right)} \\
& -2\left(1+\tilde{x}_{0}\right) \operatorname{erf}\left[\frac{\tilde{x}_{0}}{2 \sqrt{\Delta \tilde{t}}}\right]-2 \exp \left[\Delta \tilde{t}+\tilde{x}_{0}\right] \\
& \times \operatorname{erfc}\left[\frac{2 \Delta \tilde{t}+\tilde{x}_{0}}{2 \sqrt{\Delta \tilde{t}}}\right] .
\end{aligned}
$$

Its random step to be taken from the distribution of its possible displacements is sufficiently well characterized by a mean displacement, $f_{1} \sqrt{D \Delta t}$, plus a random displacement with a known root-mean-square value, $f_{2} \sqrt{D \Delta t}$,

$$
\Delta X=f_{1}\left(\frac{X}{\sqrt{D \Delta t}}, \frac{\Delta t}{t_{\text {char }}}\right) \sqrt{D \Delta t}+f_{2}\left(\frac{X}{\sqrt{D \Delta t}}, \frac{\Delta t}{t_{\text {char }}}\right) \sqrt{D} \Delta U .
$$

Here $\Delta U$ is a stochastic increment with mean zero and variance $\Delta t$, which is not necessarily Gaussian. For generating the results presented in this paper we used a very simple expression for $\Delta U$; a distribution consisting of two delta peaks with equal weights. The functions $f_{1}$ and $f_{2}$ are related to the moments of the displacement via

$$
\begin{gathered}
f_{1}\left(\frac{X}{\sqrt{D \Delta t}}, \frac{\Delta t}{t_{\text {char }}}\right)=\frac{1}{\sqrt{D \Delta t}}\left[\frac{\left\langle x^{1}\right\rangle}{\left\langle x^{0}\right\rangle}-x_{0}\right], \\
f_{2}\left(\frac{X}{\sqrt{D \Delta t}}, \frac{\Delta t}{t_{\text {char }}}\right)=\frac{1}{\sqrt{D \Delta t}} \sqrt{\frac{\left\langle x^{2}\right\rangle}{\left\langle x^{0}\right\rangle}-\left[\frac{\left\langle x^{1}\right\rangle}{\left\langle x^{0}\right\rangle}\right]^{2}} .
\end{gathered}
$$

In these expressions the factors $\left\langle x^{0}\right\rangle$ correct for the fact that a free particle may stick to the wall, so that its probability distribution is not normalized, and averages have to be corrected for this effect. Although there are four variables in the problem $\left(D, F_{\text {wall }}, x_{0}\right.$, and $\left.\Delta t\right)$, the scaling shows that any situation can be characterized by a proper scaling of $f_{1}$ and $f_{2}$, which are dimensionless functions of two variables only. For the case of a Brownian particle in free space, the values of these functions are $f_{1}=0$ and $f_{2}=\sqrt{2}$, respectively. In Fig. 4 we plotted these functions versus the scaled initial position, $x_{0} / \sqrt{D \Delta t}$, for different values of $\Delta t / t_{\text {char }}$. One sees that for large values of this ratio the wall appears almost fully reflective over the course of the time step [compare with Fig. 3 in Ref. [1]]. This is caused by the fact that the average residence time near the wall due to its stickiness is much smaller than the chosen total time step. However, for very small values of the time step, the wall behaves much more like an absorbing wall (compare with Fig. 1 in Ref. [1]).

Substitution of the analytic expressions given by Eq. (18) into Eq. (20) gives rather complicated expressions. In the simulations we use a interpolation function for $f_{1}()$ and $f_{2}()$ as function of the distance to the wall, for fixed $\Delta t$. Only distances up to $\sqrt{5 D \Delta t}$, are treated that way. Beyond this distance the particles do not feel a noticeable influence of the wall during a time step. The Brownian dynamics algorithm makes use of these interpolation functions and is almost as efficient as a conventional bulk Brownian dynamics simulation. It has $O(\Delta t)$ global discretization errors instead of $O(\sqrt{\Delta t})$ errors for a naive treatment of the wall, notwithstanding the fact that a naive treatment of a sticky wall is quite difficult to establish.

\section{AN EXAMPLE}

As an example we consider a system of particles in a one-dimensional box, of which one wall is sticky while the other one is fully reflecting. At the start of the simulation the particles are homogeneously distributed over the box and none of the particles is sticking to the sticky wall. The time dependence of the fraction of the particles that sticks to the wall is then monitored.

As mentioned before, in the simulations we use $k T=1$ and $D=1$. The only nondetermined dimension left is the length scale. We fix this length scale by choosing $F_{\text {wall }}=0$, which gives $x_{\text {char }}=1$ (and because $D=1$ also $t_{\text {char }}=1$ ). Note that the choice $F_{\text {wall }}=0$ does not mean that the wall is not "sticky." A nonsticky wall corresponds to $F_{\text {wall }}=\infty$. The system is know characterized by one free parameter, $L$, which is the distance between the two walls.

The evolution of the system proceeds in two steps. First the region near the wall is depleted. There will be a quasistationary equilibrium between the number of particles sticking to the wall and the concentration profile near the wall. This equilibrium will be established in a time proportional to $t_{\text {char }}$, the "retention time" of the particles near the sticky wall. Then the bulk concentration profile will slowly adapt to the fast dynamic equilibrium near the wall. At a certain distance from the sticky wall (a few $x_{\text {char }}$ ), the density profile of the bulk will equilibrate toward a uniform distribution. In a box of macroscopic dimensions the characteristic time for this latter process will be much larger than that for attainment of the dynamic equilibrium near the sticky wall, as it will be related to the size of the box. Clearly, this time scale will be of the order of $L^{2} / D$, where $L$ is the size of the box.

The results of this simulation are plotted in Fig. 5. The box size is chosen as $L=3$. The equilibrium fraction of particles sticking to the wall will then be

$$
\phi_{\mathrm{wall}}^{\mathrm{EQ}}=\frac{\exp \left[-F_{\text {wall }}\right]}{\exp \left[-F_{\text {wall }}\right]+L}=\frac{1}{4}
$$

This is indeed the limiting value obtained by the simulations.

The simulations were performed for three different values of the time step; $\Delta t=10^{-1}, 10^{-2}$, and $10^{-3}$ and were taken over an ensemble of $10^{6}$ particles. It is clear that the time discretization error is small, since the curves almost superimpose. The second graph in Fig. 5 shows the evolution of the average value of $x$ coordinate of the free particles. This illustrates the depletion of the region near the wall. Over 


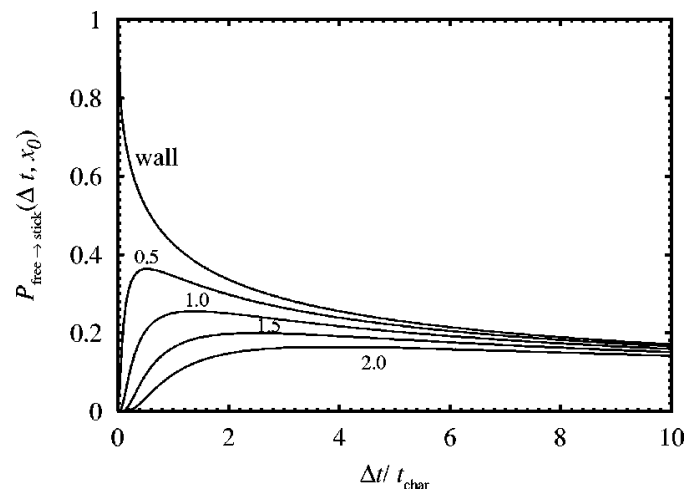

FIG. 1. The sticking probabilities for a particle that is initially at the wall and for particles that are initially free, at different distances from the wall $\left(x_{0} / x_{\text {char }}=0.5,1.0,1.5,2.0\right)$.

time the remaining free particles establish a homogeneous distribution over the box again. Therefore not only at the start, but also in the end the concentration profile of the free particles is uniform, their average position being 1.5.

\section{DISCUSSION AND CONCLUSIONS}

In this paper we presented a way to describe "sticky boundaries" that is very useful for Brownian dynamics simulations, and possibly also for other mesoscopic simulation techniques. The "sticky property" is described by a deep and steep potential well, adjacent to a reflecting boundary.

We have chosen to remain in the Brownian regime. This means that the real potential, which we model, varies slowly at the scale of the particle's free path. Therefore the particle trajectory has a fractal (random walk) nature everywhere, and the crossing of the potential barrier will not be of a ballistic nature. We think this is the correct limit for the treatment of many boundary interactions in liquids and soft condensed matter. In this limit the scaling of the characteristic time, given by Eq. (10), is different from the well-known Arrhenius scaling (which denotes a ballistic crossing of the boundary when the momentum is large enough).

For the boundary to be "sticky" only when in contact with the particle, we have studied the appropriate limiting case, where the width of the potential well approaches zero and its depth becomes infinite, in such a way that the free energy associated with this potential is kept constant. This is a purely mathematical simplification. We stay in the Brownian regime even though we approximate the barrier by an infinitely steep potential. The fact that this limit can be taken is essential for the coarse graining approach inherent to these simulations. If the width of the potential would be kept finite, one would introduce a very small time scale into the system (namely, the diffusion time corresponding to that width). In simulations this time scale must then be resolved. This would limit the discretization time step to very small values. In our approach this problem is avoided.

Indeed, the limiting case of a zero width of the potential well is in the spirit of the Brownian dynamics method. In this method the correlation time of momentum equilibration of particles is also taken to be zero. As a result, the smallest
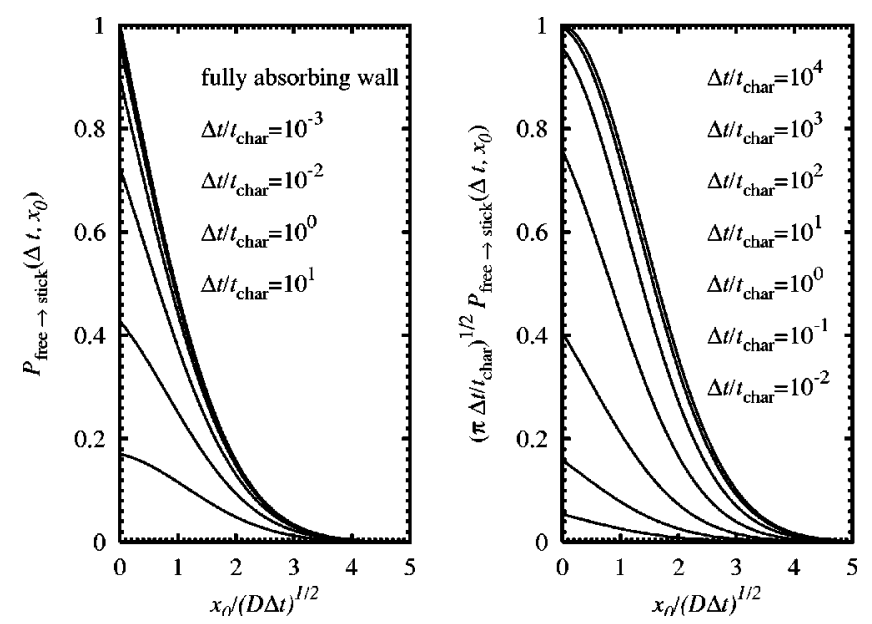

FIG. 2. The position dependence of the sticking probability $P_{\text {free } \rightarrow \text { stick }}\left(\Delta t, x_{0}\right)$ for several values of $\Delta t$, scaled in two ways, corresponding to its short (left) and long (right) time behavior. The order of the curves is the same as the order of the labels next to them.

remaining characteristic time scale is the time needed to diffuse over the next smallest characteristic length scale in the system. In most cases this allows for using time steps that are orders of magnitude larger than those used in simulation techniques in which the full Newtonian equations are solved, such as molecular dynamics. The process introduced in this paper therefore constitutes a course graining of the short range interactions, by setting the time scales associated with these interactions equal to zero.

Note that this way of course graining, which we would like to call "hardening coarse graining," in a sense is opposite to coarse graining procedures that are commonly used. In many methods, such as the dissipative particle method [2],

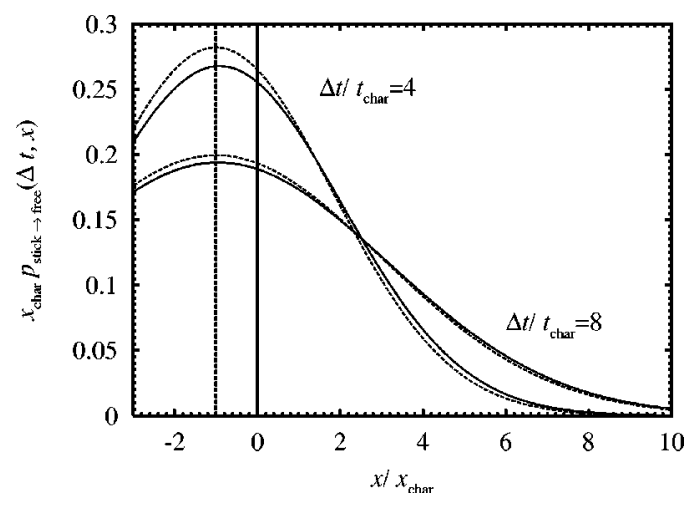

FIG. 3. The probability density for a particle that is initially sticking to the wall is given by the solid lines. The sticky wall is positioned at $x=0$ (solid vertical line), so the values to the left of this point have no real physical meaning. The long time behavior of a particle starting at this sticky wall is equivalent to that of a freely diffusing particle (no energy barrier), starting from a reflecting wall, which is positioned at $x=-x_{\text {char }}$ (dashed vertical line). Note that the original wall now only indicates the origin of the position axis but has no physical "wall properties" anymore. In this case the probability density would be a reflected Gaussian with its center at $x=-x_{\text {char }}$. This curve is given by the dotted lines. 

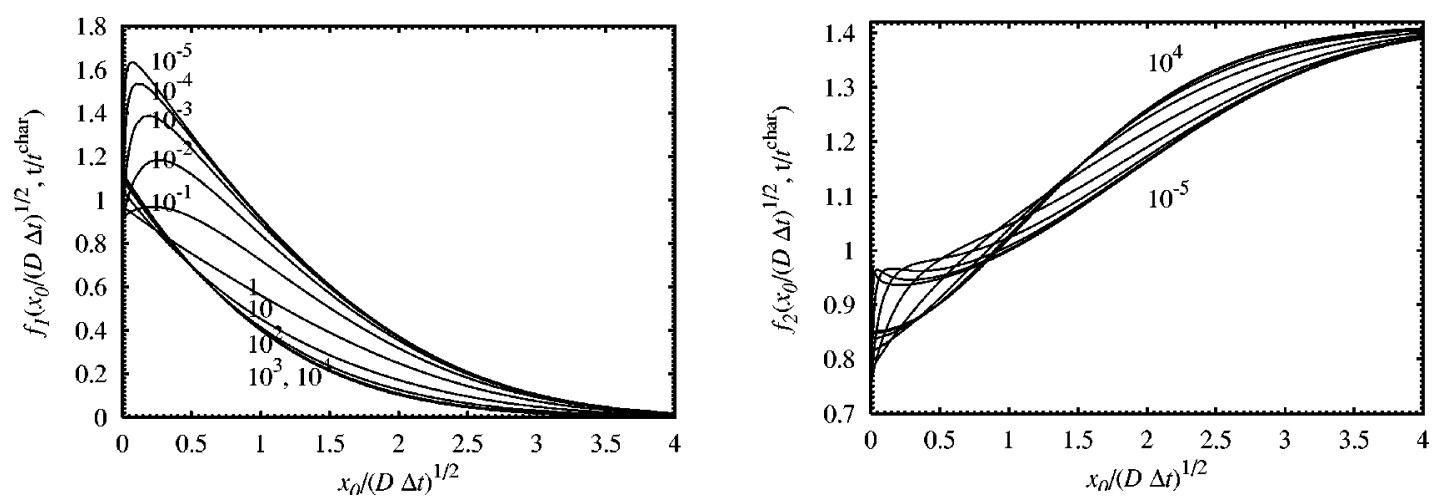

FIG. 4. The dimensionless mean displacement $f_{1}$ and the root-mean-square displacement $f_{2}$ [see Eq. (20)], versus the scaled initial distance from the sticky wall, for several values of $\Delta t / t_{\text {char }}\left(10^{-5}\right.$ to $\left.10^{4}\right)$. For time steps much smaller than the characteristic or retention time the wall behaves as an absorbing wall, whereas for much larger time steps it behaves as a reflecting wall.

one introduces "effective particles," which consist of a number of physical particles and which have a larger size. The interactions between these particles are averaged, softer, than those between the constituent, original particles. This treatment is what we would like to call "softening coarse graining." It means that the time scales associated with interactions are made larger in this procedure (instead of being put to zero). This softening causes all "hardness" to disappear from the coarse-grained system. For some systems this does not invoke problems, but for others it means that essential features might be lost in the process. Important examples of the latter case are systems with topological constraints such as glasses and entangled polymer systems. We expect that a "good" coarse graining treatment of such systems needs both kinds of coarse graining. Some interactions, the more local ones, can be replaced by interactions with time scale zero, such as used in this paper, others, longer ranged interactions, probably should become softer in the appropriate coarse graining process. Therefore, in a way, the method presented in this paper can be considered a next step in the process of progressive coarse graining operations.

The sticky boundary introduced in the current paper is a

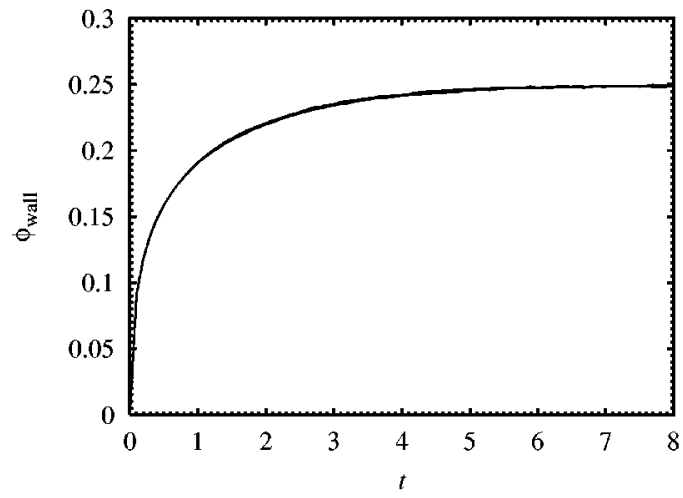

very "pure boundary" in the sense that it is characterized by one fundamental parameter only, namely, the "wall free energy," which is free from "local features" such as the precise shape of the potential. Therefore we expect our approach to have several attractive applications. The most trivial one is a proper description of interactions of Brownian particles with a "sticky" wall. After connecting a number of these particles by springs, one can study the interaction of a polymer with a "sticky" wall. The situation becomes even more interesting if the particles themselves are made sticky. This opens up the possibility to correctly simulate solvent quality in a polymer solution, using pure Brownian dynamics simulations and very short range interactions. A way to do this is to use particles with a certain volume and make their surface sticky.

If different beads are given different sticky properties, one can simulate surfactant systems. Maybe even the main features of proteins, containing chemical groups exhibiting all kinds of mutual (local) interactions, can be modeled in this way. Of course, some issues have to be resolved first. One issue is the difference between a flat wall and a curved bead. Another one is how to properly treat particles that stick together in clusters.

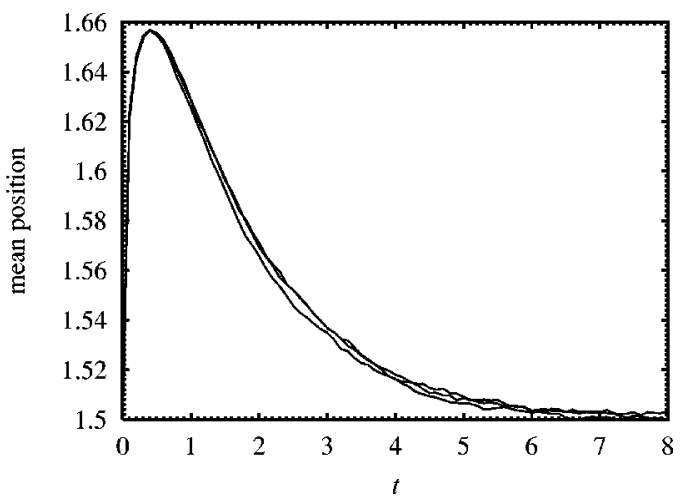

FIG. 5. The left graph shows the time evolution of the fraction of particles at the sticky wall, in a box of size $L=3$, having one sticking wall and one reflecting wall. The simulation was started with a homogeneous distribution of $10^{6}$ particles, none of them sticking to the wall. For the parameters we chose $F_{\text {wall }} / k T=0$ and $D=1$. The three almost identical curves correspond to different choices for the time step $\left(\Delta t=10^{-1}, 10^{-2}\right.$, and $\left.10^{-3}\right)$. In the right graph the time evolution of the mean position of the particles in the bulk is shown. This demonstrates the initial depletion of the region near the wall, leading to a mean value for the $x$ coordinate of the free particles larger than $L / 2$. Over time, the remaining free particles establish a homogeneous distribution over the box again. 
Approaching the end of this paper we would like to stress again that the method we introduced here fully relies on the fundamental idea that one can perform accurate Brownian dynamics simulations if only the zeroth, first, and second moments of the probability distribution of the particle displacement are sampled correctly. This idea was introduced in Ref. [1]. In any case it is clear that it would have been virtually impossible to obtain the results presented here by only using a "naive algorithm" to treat the particle behavior near the "sticky" boundary. This would have meant introducing the very small time scale discussed above.

\section{ACKNOWLEDGMENT}

The research of Dr. Peters has been supported by the Royal Netherlands Academy of Arts and Sciences.

\section{APPENDIX A: THE GREEN FUNCTION FOR A SQUARE POTENTIAL WELL NEXT TO A REFLECTING WALL}

In this appendix we consider the problem of a diffusing particle in a square potential well adjacent to a wall. Generally, this problem is described by

$$
\frac{\partial p}{\partial t}=-\boldsymbol{\nabla} \cdot \boldsymbol{j},
$$

where $p$ is the probability distribution (the "concentration") of the particle and $\boldsymbol{j}$ is its probability flux (the "flow density"). In dimensionless, one-dimensional, form we obtain,

$$
\frac{\partial}{\partial t} p(x, t)=(\mathcal{L} p)(x, t),
$$

where $\mathcal{L}$ is the operator

$$
(\mathcal{L} p)(x, t)=\frac{\partial}{\partial x}\left[p(x, t) \frac{\partial}{\partial x} U(x)+\frac{\partial}{\partial x} p(x, t)\right],
$$

where the divergence has become a simple derivative, and the (negative of the) flux is given by the two contributions between the square brackets. The first term stems from the force on the particle exerted by the potential field, the second gives the (pure) diffusion contribution, due to the probability ("concentration") gradient.

We now assume that the particle resides on the positive $x$ axis. At $x=0$ we position an impenetrable, reflecting wall. Further, the dimensionless potential $U(x)$ is taken to be a square potential,

$$
U(x)= \begin{cases}-E & \text { for } x<b, \\ 0 & \text { for } x>b .\end{cases}
$$

The formal solution of this problem is given by

$$
p(x, t)=\int_{0}^{\infty} G\left(t, x, x_{0}\right) p\left(x_{0}, 0\right) d x_{0} .
$$

Further on we show that this formal expression appears naturally. For our aim of describing the motion of the Brownian particle over the period of a time step, Eq. (A5) is very useful. Since we know the initial position, $x_{0}$, of the particle, its probability distribution at $t=0$ is given by a delta function. This means that in the end the formal expression will reduce to the desired form,

$$
p(x, t)=G\left(t, x, x_{0}\right) .
$$

We return to this point later.

To solve Eq. (A2) we note that it is linear in the probability density $p$. We can therefore apply the method of separation of variables. First we assume that the solution for $p$ can be written as a product of time and position dependent functions, e.g., $p(x, t)=\psi(t) \phi(x)$. We substitute this solution and then divide both sides of Eq. (A2) by this product. This gives

$$
\frac{1}{\psi_{\lambda}} \frac{\partial \psi_{\lambda}}{\partial t}=\frac{1}{\phi_{\lambda}} \mathcal{L} \phi_{\lambda} \equiv-\lambda^{2}
$$

The right- and left-hand sides each depend on a different, independent variable. Both terms must therefore be equal to some constant. Due to the diffusion process, the probability distribution must "spread," e.g., its time derivative must be negative. We used this "physical intuition" to introduce the square and the minus sign. The subscript $\lambda$ takes into account that for every possible value of the separation constant $\lambda$ we obtain a different, valid solution. For the time dependent factor in $p(x, t)$ we find immediately

$$
\psi(t) \sim \exp \left[-\lambda^{2} t\right] .
$$

For the position dependence we must solve the other part of Eq. (A7), e.g., construct the eigenfunctions of the operator $\mathcal{L}$, given by Eq. (A3),

$$
\mathcal{L} \phi_{\lambda}=-\lambda^{2} \phi_{\lambda} .
$$

If the operator $\mathcal{L}$ is self-adjoint, one obtains a complete set of orthogonal eigenfunctions $\left\{\phi_{\lambda}\right\}$. In this case one can use the theory of self-adjoint operators to form the Green function using the eigenfunctions. For arbitrary $U(x)$ the operator $\mathcal{L}$ is not self-adjoint for the ordinary functional inner product. Nevertheless, one can easily find a generalized inner product [given by Eq. (A21)], such that the operator is self-adjoint for this inner product and the theory is applicable. Having noted this we can safely proceed.

The potential $U(x)$ is flat, except for the jump at $x=b$. This means that in both areas separated by this jump this operator reduces to a simple second derivative. The eigenfunctions of $\mathcal{L}$ are therefore ordinary sines and cosines, which have to be correctly matched at the jump. For the interval $[0, b]$ the appropriate solution must be a cosine, because of the reflecting boundary condition, implying a zero probability flux on the wall. Therefore, we find as a general solution to Eq. (A9), 


$$
\phi_{\lambda}(x)= \begin{cases}\cos \lambda x & \text { for } x<b, \\ \alpha \cos \lambda x-\beta \sin \lambda x & \text { for } x>b\end{cases}
$$

The appropriate choices for $\alpha$ and $\beta$ are found by considering the probability flux $j(x)$ at the jump of the potential,

$$
j(x)=-p(x) \frac{\partial}{\partial x} U(x)-\frac{\partial}{\partial x} p(x) .
$$

Integrating Eq. (A1) over a very small interval $[A, B]$, around the jump in the potential $(A<b<B)$, we obtain an expression for the time derivative of the number of particles in this interval.

$$
\frac{\partial}{\partial t} \int_{A}^{B} p(x) d x=-\int_{A}^{B} \frac{\partial j}{\partial x} d x=j(A)-j(B) .
$$

It is clear that if $B-A \rightarrow 0$ (keeping $A<b<B$ ), the left-hand side of this expression must become zero. Otherwise particles would suddenly "disappear" at the potential jump. The right-hand side then tells us that $j$ must be continuous. As $j$ contains at least one $\delta$-function [the derivative of a step function, cf. Eqs. (A4) and (A11)], the probability density $p$ should also contain a step function, to cancel this singularity. Assuming that the change in the probability density on both sides of the potential jump will be governed by Boltzmann's law, we propose for the probability density a form given by

$$
p(x)=f(x) \exp [-U(x)],
$$

where $f(x)$ is a continuous function of $x$. Substitution in Eq. (A11) using Eq. (A4) gives

$$
\begin{aligned}
j(x)= & -f \exp [-U(x)] E \delta(x-b)-\frac{\partial f}{\partial x} \exp [-U(x)] \\
& +f \exp [-U(x)] E \delta(x-b)=-\frac{\partial f}{\partial x} \exp [-U(x)] .
\end{aligned}
$$

Indeed, this removes the $\delta$ function singularity in $j(x)$, as $f$ is continuous. Further, $j$ will be continuous in $x=b$, provided that

$$
\lim _{x \uparrow b} \frac{\partial f}{\partial x} \exp [E]=\lim _{x \downarrow b} \frac{\partial f}{\partial x}
$$

Applying these conditions, for every eigenfunction individually, one finds that

$$
\begin{gathered}
\lim _{x \uparrow b} \exp [-E] \phi_{\lambda}(x)=\lim _{x \downarrow b} \phi_{\lambda}(x), \\
\lim _{x \uparrow b} \frac{\partial}{\partial x} \phi_{\lambda}(x)=\lim _{x \downarrow b} \frac{\partial}{\partial x} \phi_{\lambda}(x) .
\end{gathered}
$$

After substitution of Eq. (A10) and solving the equations for $\alpha$ and $\beta$, one finds $\phi_{\lambda}(x)$

$$
= \begin{cases}\cos \lambda x & \text { for } x<b, \\ \left(\sin ^{2} \lambda b+\cos ^{2} \lambda b \exp [-E]\right) \cos \lambda x & \\ -\sin \lambda b \cos \lambda b(1-\exp [-E]) \sin \lambda x & \text { for } x>b .\end{cases}
$$

Using straightforward trigonometry rules one can obtain another equivalent expression for the case $x>b$,

$$
\begin{aligned}
\phi_{\lambda}(x)= & \left(\frac{1}{2}+\frac{1}{2} \exp (-E)\right) \cos \lambda x \\
& -\left(\frac{1}{2}-\frac{1}{2} \exp (-E)\right) \cos \lambda(2 b-x) .
\end{aligned}
$$

Now we can write the general solution for $p(x, t)$, as a superposition of all solutions for the possible $\lambda$ values,

$$
p(x, t)=\int_{0}^{\infty} C(\lambda) \phi_{\lambda}(x) \exp \left(-\lambda^{2} t\right) d \lambda,
$$

where $C(\lambda)$ gives the contribution of every eigenfunction to the final solution. Its precise form is determined by the initial condition, as we will see. Setting $t=0$ we find

$$
p(x, 0)=\int_{0}^{\infty} C(\lambda) \phi_{\lambda}(x) d \lambda .
$$

The precise form of $C$ can now be found by taking the proper functional inner product involving $p(x, 0)$ and the set of eigenfunctions. One should note that the operator $\mathcal{L}$ is not self-adjoint for the usual functional inner product, but it is easy to show it has this property, $(\mathcal{L} f, g)=(f, \mathcal{L} g)$, for the generalized inner product with the proper weight function, defined by (see Ref. [3])

$$
(f, g)=\int_{0}^{\infty} \exp [U(x)] f(x) g(x) d x,
$$

where $f$ and $g$ obey the same, homogeneous boundary conditions. The generalized inner product, Eq. (A21), of two eigenfunctions is then given by

$$
\begin{aligned}
\left(\phi_{\lambda}, \phi_{\lambda^{\prime}}\right) & =\frac{\pi}{2}\left(\sin ^{2} \lambda b+\exp [-2 E] \cos ^{2} \lambda b\right) \delta\left(\lambda-\lambda^{\prime}\right) \\
& \equiv A(\lambda) \delta\left(\lambda-\lambda^{\prime}\right)
\end{aligned}
$$

Now we can determine $C(\lambda)$, by taking a functional inner product of Eq. (A20) with an eigenfunction, 


$$
\begin{aligned}
\int_{0}^{\infty} \exp [U(x)] \phi_{\lambda^{\prime}}(x) p(x, 0) d x \\
\quad=\int_{0}^{\infty} C(\lambda) \int_{0}^{\infty} d x \exp [U(x)] \phi_{\lambda^{\prime}}(x) \phi_{\lambda}(x) d \lambda \\
\quad=\int_{0}^{\infty} C(\lambda) A(\lambda) \delta\left(\lambda-\lambda^{\prime}\right) d \lambda=A\left(\lambda^{\prime}\right) C\left(\lambda^{\prime}\right) .
\end{aligned}
$$

Combining Eqs. (A19), (A20), and (A23), we can write

$$
\begin{aligned}
p(x, t)= & \int_{0}^{\infty} \frac{\int_{0}^{\infty} d x^{\prime} \exp \left[U\left(x^{\prime}\right)\right] \phi_{\lambda}\left(x^{\prime}\right) p\left(x^{\prime}, 0\right)}{A(\lambda)} \phi_{\lambda}(x) \\
& \times \exp \left(-\lambda^{2} t\right) d \lambda,
\end{aligned}
$$

which has indeed the form of Eq. (A5), so that we can finally write the Green function as

$$
\begin{aligned}
G\left(t, x, x_{0}\right)= & \frac{2}{\pi} \int_{0}^{\infty} \exp \left[U\left(x_{0}\right)\right] \frac{\phi_{\lambda}(x) \phi_{\lambda}\left(x_{0}\right)}{\sin ^{2} \lambda b+\exp [-2 E] \cos ^{2} \lambda b} \\
& \times \exp \left(-\lambda^{2} t\right) d \lambda .
\end{aligned}
$$

\section{APPENDIX B: THE STICKY BOUNDARY CONDITION FOR THE FOKKER-PLANCK EQUATION}

Away from the "sticky" wall the Brownian particle diffuses freely. Therefore, everywhere in space the probability distribution, $p_{\text {free }}(x, t)$, should obey the Fokker-Planck equation. The wall is macroscopically occupied, with probability $P_{\text {stick }}(t)$. Particles at the wall can become free particles and vice versa. To get a closed set of equations for $p_{\text {free }}$, a boundary condition is needed that takes into account this wall interaction. The probability flux at the wall must equal the decrease per unit time of the sticking probability at the wall, thus

$$
j(t, 0)=-\frac{\partial}{\partial t} P_{\text {stick }}(t) .
$$
to

During a time step $\Delta t$ the probabilities evolve according

$$
\begin{aligned}
P_{\text {stick }}(t+\Delta t)= & P_{\text {stick }}(t) P_{\text {stick } \rightarrow \text { stick }}(\Delta t) \\
& +\int_{0}^{\infty} p_{\text {free }}\left(t, x_{0}\right) P_{\text {free } \rightarrow \text { stick }}\left(\Delta t, x_{0}\right) d x_{0},
\end{aligned}
$$

$$
\begin{aligned}
p_{\text {free }}(t+\Delta t, x)= & P_{\text {stick }}(\Delta t) p_{\text {stick } \rightarrow \text { free }}(\Delta t, x) \\
& +\int_{0}^{\infty} p_{\text {free }}\left(t, x_{0}\right) p_{\text {free } \rightarrow \text { free }}\left(\Delta t, x, x_{0}\right) d x_{0} .
\end{aligned}
$$

When trying to calculate $j(t, 0)$ by inserting Eq. (B2) into Eq. (B1) and using the detailed expressions for the transitional probabilities, one encounters difficulties. For instance, when using the small time expression Eq. (11), one gets a divergent result (as $1 / \sqrt{\Delta t}$ in the finite difference approximation). The reason for this problem is that the initial values of $P_{\text {stick }}(t)$ and $p_{\text {free }}(t, x)$ cannot be chosen independently. The divergent result indicates an infinitely fast "equilibration" of initial conditions if they do not obey the interdependence.

The correct boundary condition can be most easily derived using the jump conditions of the square well potential Eq. (A16). This condition states that an eigenfunction before and after the jump always differs a factor $\exp [-E]$. Since any solution is a sum of eigenfunctions, this means that this factor is the same for any function. Furthermore, in the limit of $b \rightarrow 0$ the well has such a small gap that it is always internally equilibrated, i.e., the probability density is constant in the well. By integrating over $x(0<x<b)$ one then finds that

$$
p_{\text {free }}(t, 0)=\exp \left[F_{\text {wall }}\right] P_{\text {stick }}(t)
$$

Combining this expression with Eq. (B1) one obtains the boundary condition for $p_{\text {free }}$,

$$
\begin{aligned}
\frac{\partial}{\partial t} p_{\text {free }}(t, 0)= & -\exp \left[F_{\text {wall }}\right] j(t, 0)=\exp \left[F_{\text {wall }}\right] \\
& \times\left\{-v p_{\text {free }}(t, 0)+D \frac{\partial}{\partial x} p_{\text {free }}(t, 0)\right\},
\end{aligned}
$$

where $v$ is a drift velocity, imposed by additional, deterministic forces, if present. Two extreme cases are the fully adsorbing and the fully reflecting boundary conditions. Fully absorbing means that $F_{\text {wall }} \rightarrow-\infty$. In this case Eq. (B3) gives that $p_{\text {free }}(t, 0)=0$ [since $P_{\text {stick }}(t)$ is bounded]. The fully reflecting limit is obtained for $F_{\text {wall }} \rightarrow \infty$, here Eq. (B4) gives that $j(t, 0)=0$.
[1] E. Peters and T. Barenbrug, preceding paper, Phys. Rev. E 66, 056701 (2002).

[2] P. Espanol and P. Warren, Europhys. Lett. 30, 191 (1995).
[3] H. Risken, The Fokker-Planck Equation: Methods of Solution and Applications (Springer-Verlag, Berlin, 1989). 